\title{
Sharper Focal Spot for a Radially Polarized Beam Using Ring Aperture with Phase Jump
}

\author{
Svetlana N. Khonina and Andrei V. Ustinov \\ Image Processing Systems Institute of the Russian Academy of Sciences, Molodogvardeiskaya Street 151, Samara 443001, Russia \\ Correspondence should be addressed to Svetlana N. Khonina; khonina@smr.ru
}

Received 28 November 2012; Revised 4 March 2013; Accepted 10 March 2013

Academic Editor: You-Rong Li

Copyright (c) 2013 S. N. Khonina and A. V. Ustinov. This is an open access article distributed under the Creative Commons Attribution License, which permits unrestricted use, distribution, and reproduction in any medium, provided the original work is properly cited.

\begin{abstract}
We study analytically and numerically in which way the width of ring aperture containing a phase jump affects the size and intensity of the focal spot generated with a radially polarized beam. It is shown that by means of destructive interference of beams coming from the different-phase rings it becomes possible to overcome the scalar diffraction limit corresponding to the first zero of the zero-order Bessel function. The minimal focal spot size $(\mathrm{FWHM}=0.33 \lambda)$ is found to be attained when the annular aperture width amounts to $20 \%$ of the full-aperture radius. In this case, the side-lobe intensity is not larger than $30 \%$ of the central peak. A wider annular aperture with the phase jump introduced is also shown to form a focal spot not exceeding the diffraction limit for a narrow annular aperture, simultaneously providing a nearly six times higher intensity. In this case, the side lobes amount to $35 \%$ of the central peak.
\end{abstract}

\section{Introduction}

A narrow annular pupil that blocks the light from propagating practically through the entire central part of the lens [1-3] is a simple, albeit low-efficiency technique to generate narrowextended beams in the focal plane.

More complicated techniques for the full-aperture apodization of the pupil's function that employ both purely phase and amplitude-phase distributions have also been reported [4-9]. In this case, a tighter focal spot is normally obtained at a sacrifice of the energy redistribution from the central peak to the side lobes. This situation is in full compliance with the Toraldo di Francia theory [10], which states that the central focal spot can be made as small as one likes but at a sacrifice of increasing side lobes, which sometimes become several times [11], or even orders $[8,9]$, larger than the central peak.

The presence of the substantial side lobes limits the use of "superresolution" elements in the imaging and optical data recording systems, in which the acceptable side-lobe level is $30 \%$ of the central peak [12].

The optimization procedures aimed at controlling the growth of the side lobes inevitably lead to a widened central spot $[12,13]$. With the side lobes' intensity being less than $30 \%$ of the central peak intensity, they can be filtered out [14] or leveled as a result of nonlinear light interaction with the recording medium [15].

The focal spot can be made tighter without an essential increase in the side lobes by introducing a radial phase jump which leads to the destructive interference between two light beams generated by each of the pupil's rings $[15,16]$. The destructive action of the $\pi$-phase jump is well known in the coding methods also $[17,18]$.

Note that although the full-aperture apodization of the lens pupil with binary radial elements is usually considered $[4,6-8,16,19-21]$, it has been numerically demonstrated [22] that by diaphragming the lens central part ( $75 \%$ in terms of radius) the focus can be made considerably tighter.

In this paper, we study analytically and numerically in which way the size of an annular pupil with a radial phase jump affects the focal spot size generated by a radially polarized beam. It has been shown that the destructive interference between the beams passing different-phase rings enables going beyond a scalar limit corresponding to the zero-order Bessel function (FWHM $=0.36 \lambda)$. A tighter focal spot $(\mathrm{FWHM}=0.33 \lambda)$ is attained at a sacrifice of a lower 
focal intensity and higher side lobes that corresponds to the Toraldo di Francia theory. However, due to increase of width of the ring aperture, we can increase energy in the central spot and keep a level of side lobes below $30 \%$ of the central peak.

The analysis of the sharp focusing based on the RichardsWolf integrals [23] has shown that the dependence of the focal spot size on the width of the narrow annular pupil is different for the scalar and vector models. In the former case, the diffraction-limited focal spot size is attained when the width of the ring that transmits the peripheral rays tends to zero, while the focal intensity drops quadratically with decreasing width. In the latter case, the minimal focal spot size is attained at a fixed annular pupil width, with the focal intensity being proportional to the ring width to the $(3 / 4)$ th power.

The numerical simulation has shown that notwithstanding the destructive interference, by choosing a fairly wide annular pupil, the focal point intensity can be essentially increased without exceeding the limit corresponding to the narrow annular pupil.

\section{Analysis of Generating a Sharper Focal Spot: Scalar Model}

The focusing of a bounded plane wave with a lens of radius $R$ and focal length $f$ is described by a Fourier-Bessel transform, with the intensity in the focal plane given by

$$
I_{w}(\rho)=\left|\frac{k}{f} \int_{0}^{R} J_{0}\left(\frac{k \rho r}{f}\right) r \mathrm{~d} r\right|^{2}=\left|\frac{R}{\rho} J_{1}\left(\frac{k \rho r}{f}\right)\right|^{2},
$$

which describes an Airy pattern generated in the lens focal plane.

Formally, obtaining a Bessel beam in the focal plane requires taking a Fourier-Bessel transform of an infinitely narrow annular pupil:

$$
\begin{aligned}
I_{\delta}(\rho) & =\left|\frac{k}{f} \int_{0}^{\infty} \delta\left(\frac{r}{R}-1\right) J_{0}\left(\frac{k \rho r}{f}\right) r \mathrm{~d} r\right|^{2} \\
& =\left|\frac{k R^{2}}{f} J_{0}\left(\frac{k \rho R}{f}\right)\right|^{2} .
\end{aligned}
$$

The numerical aperture (NA) of the lens of radius $R$ and focal length $f$ is defined by the following relationship:

$$
\mathrm{NA}_{\text {lens }}=n \sin \theta=n \sin \left[\operatorname{arctg} \frac{R}{f}\right]
$$

or, in the paraxial case,

$$
\mathrm{NA}_{\text {lens }} \approx \frac{n R}{f},
$$

where $n$ is refractive index of media.

The resulting focal spot size in the cases under study is characterized by the following parameters.

When focusing on the full aperture of (1), the central focal spot radius is determined by the first zero of the first-order Bessel function $\left(J_{1}\left(\gamma_{11}\right)=0, \gamma_{11}=3.83\right)$ :

$$
\rho_{\text {lens }}=\frac{3.83 \lambda f}{2 \pi R}=0.61 \frac{\lambda f}{R} \text {. }
$$

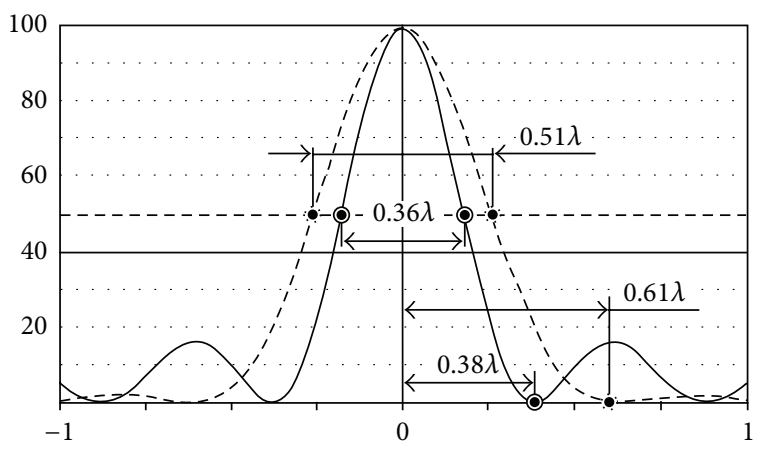

FIGURE 1: Illustration of the focal spot for the full-aperture illumination and with narrow ring $(R=f)$.

In the paraxial case,

$$
\rho_{\text {lens }} \approx \frac{0.61}{\mathrm{NA}} \lambda .
$$

The estimate for the focal spot size at half level of maximum intensity is

$$
\mathrm{FWHM}_{\text {lens }}=0.515 \frac{\lambda f}{R}
$$

and for the spot area is

$$
\mathrm{HMA}_{\text {lens }}=\pi \cdot\left(\frac{\mathrm{FWHM}_{\text {lens }}}{2}\right)^{2} \approx 0.21\left(\frac{\lambda f}{R}\right)^{2} .
$$

When focusing on the narrow annular diaphragm of (2), the focal spot radius is determined by the first zero of the zeroorder Bessel function $\left(J_{0}\left(\gamma_{01}\right)=0, \gamma_{01}=2.405\right)$ :

$$
\rho_{\text {ring }}=\frac{2.4 \lambda f}{2 \pi R}=0.38 \frac{\lambda f}{R} .
$$

In the paraxial case,

$$
\rho_{\text {ring }} \approx \frac{0.38}{\mathrm{NA}} \lambda .
$$

The comparison of (5) and (9) suggests that introducing the narrow ring enables generating a 1.6 times smaller focal spot even at high NA (Figure 1).

The estimate for the focal spot size at half level of maximum intensity is

$$
\mathrm{FWHM}_{\text {ring }}=0.357 \frac{\lambda f}{R},
$$

which is 1.44 times lower than for the full-aperture illumination, whereas the spot area at half-maximum intensity is defined as

$$
\mathrm{HMA}_{\text {ring }}=\pi \cdot\left(\frac{\mathrm{FWHM}_{\text {ring }}}{2}\right)^{2}=0.1\left(\frac{\lambda f}{R}\right)^{2},
$$

which, accordingly, is 2.1 times smaller. 
Note, however, that (2) disregards the amplitude decrease associated with increasing illuminated area, as the value of the $\delta$-function is assumed to be equal to infinity.

Assume that the ring is not infinitely narrow, as is the case in (2), but of a finite width that comprises a small fraction $\Delta$ of the lens radius. In this case, the complex amplitude is given by

$$
E_{\Delta}(\rho)=\frac{k}{i f} \exp (i k f) \int_{R(1-\Delta)}^{R} J_{0}\left(\frac{k r \rho}{f}\right) r \mathrm{~d} r .
$$

In view of the small $\Delta$, the integral (13) can be approximately calculated by multiplying the integrand value at the middle point:

$$
r_{s}=R\left(1-\frac{\Delta}{2}\right)
$$

by the line length

$$
\begin{aligned}
E_{\Delta}(\rho)= & \frac{k}{i f} \exp (i k f) \\
& \times\left[R^{2} \Delta\left(1-\frac{\Delta}{2}\right) J_{0}\left(\frac{k \rho R(1-\Delta / 2)}{f}\right)\right] \\
\approx & \frac{k R^{2} \Delta}{i f} \exp (i k f) J_{0}\left(\frac{k \rho R}{f}\right) .
\end{aligned}
$$

We can see that although this approximation gives the same focal spot size as that for the infinitely narrow ring (2), this relation also takes into account that the amplitude decreases at the center proportionally to the decreasing annular aperture width $\Delta$. If $R(1-\Delta / 2)$ is not replaced with $R$ in the Bessel function argument, the spot radius estimate becomes a bit larger.

Below, we discuss in which way the focal spot size can be made smaller by introducing a phase jump of $\pi$-radian along the central radius of the narrow annular aperture:

$$
\tau(r)= \begin{cases}0, & 0 \leq r<R(1-\Delta), \\ 1, & R(1-\Delta) \leq r<R\left(1-\frac{\Delta}{2}\right), \\ \exp (i \pi), & R\left(1-\frac{\Delta}{2}\right) \leq r \leq R .\end{cases}
$$

A radial phase jump which leads to the destructive interference between two light beams generated by each of the pupil's rings was used for sharp focusing $[15,16]$. The destructive action of the $\pi$-phase jump is well known in the coding methods also $[17,18]$.
The resulting distribution in the focal spot will correspond to the destructive interference of beams generated by the individual rings:

$$
\begin{aligned}
& E_{\tau}(\rho) \\
& =\frac{k}{i f} \exp (i k f) \int_{0}^{R} \tau(r) J_{0}\left(\frac{k r \rho}{f}\right) r \mathrm{~d} r \\
& =\frac{k}{i f} \exp (i k f) \\
& \quad \times\left[\int_{R(1-\Delta)}^{R(1-\Delta / 2)} J_{0}\left(\frac{k r \rho}{f}\right) r \mathrm{~d} r-\int_{R(1-\Delta / 2)}^{R} J_{0}\left(\frac{k r \rho}{f}\right) r \mathrm{~d} r\right] .
\end{aligned}
$$

In view of (15), we obtain

$$
\begin{aligned}
E_{\tau}(\rho)=\frac{\Delta}{2} & \frac{k R^{2}}{i f} \exp (i k f) \\
\times & {\left[J_{0}\left(\frac{k \rho R(1-3 \Delta / 4)}{f}\right)\left(1-\frac{3 \Delta}{4}\right)\right.} \\
& \left.\quad-J_{0}\left(\frac{k \rho R(1-\Delta / 4)}{f}\right)\left(1-\frac{\Delta}{4}\right)\right] .
\end{aligned}
$$

From (18) it is clear that in spite of destructive interference there is any nonzero intensity at the focal point because the inner and outer areas of the annular aperture are not equal.

To estimate the resulting equation (18) for small $\rho$, let us recall that the Bessel function can be approximately expressed through a Taylor series [24]:

$$
\begin{aligned}
J_{\nu}(x \longrightarrow 0) \sim & \frac{1}{\Gamma(v+1)}\left(\frac{x}{2}\right)^{\nu} \\
& \times\left[1-\frac{x^{2}}{4(v+1)}+\frac{x^{4}}{32(v+1)(v+2)}-\cdots\right] .
\end{aligned}
$$

Then, (18) is approximately reduced to

$$
\begin{aligned}
E_{\tau}(\rho) \approx & -\left(\frac{\Delta}{2}\right)^{2} \frac{k R^{2}}{i f} \exp (i k f) \\
& \times\left[1-\left(\frac{k \rho R}{2 f}\right)^{2}\left(3-\frac{15}{8} \Delta+\frac{13}{16} \Delta^{2}\right)\right] .
\end{aligned}
$$

The first coefficient in the right-hand side of (20) shows that the central spot amplitude falls proportionally to the annular aperture width squared. This effect is due to the fact that the radius of $\pi$-phase jump is the middle of the ring. The negative value at the zero point means that there is a phase shift by $\pi$ with respect to the incident beam.

The focal spot radius is derived by setting the expression in the square brackets equal to zero, so that

$$
\rho_{\text {jump }}=\frac{\lambda f}{\pi R \sqrt{3-(15 / 8) \Delta+(13 / 16) \Delta^{2}}} .
$$


Because $\Delta<1$, the smallest radius in (21) is attained at $\Delta \rightarrow 0$, being equal to

$$
\rho_{\text {min } \_a}^{\text {jump }}=\frac{\lambda f}{\pi R \sqrt{3}} \approx 0.184 \frac{\lambda f}{R} .
$$

Note that the estimate in (22) is understated since with the quadratic approximation of the Bessel function in (19), the resulting value appears to be $20 \%$ lower than the true value. Therefore, we will consider the following value as more accurate:

$$
\rho_{\min }^{\text {jump }}=1.2 \rho_{\min \_a}^{\text {jump }}=0.22 \frac{\lambda f}{R}
$$

This estimate predicts that the radius should be 2.8 times smaller than for the full-aperture lens and 1.7 times smaller than for the narrow annular aperture.

The estimates for the focal spot diameter and area at halfmaximum intensity are as follows:

$$
\begin{gathered}
\mathrm{FWHM}_{\text {min }}^{\text {jump }}=0.26 \frac{\lambda f}{R}, \\
\mathrm{HMA}_{\text {min }}^{\text {jump }}=0.053\left(\frac{\lambda f}{R}\right)^{2} .
\end{gathered}
$$

Note that, in this case, the focal spot diameter FWHM in (24) appears to be larger than the radius measured as far as the zero-intensity point (23). This situation differs from the one occurred for the first- and zero-order Bessel functions, for which the spot size FWHM was smaller than the zero-intensity radius, showing that the central peak walls are steeper.

\section{Analysis of the Focal Spot Size Decrease When a Radially Polarized Beam Is Tightly Focused}

When doing the tight focusing of a radially polarized beam with the use of a narrow annular pupil, the major contribution to the focal plane has been known to be from the longitudinal E-field component [1-3]. The significant predominance of one component over the other vector field components creates a situation similar to the scalar regime. Thus, scalar theory instruments can be successfully utilized, producing fairly accurate results. The tightly focused focal spot usually appears to be larger in size than the scalar estimate as different components really contribute to the focal spot.

Regarding the above considerations, only the longitudinal component will be taken into account when analyzing the tight focusing of radially polarized light.

For a narrow annular pupil, we obtain [9]

$$
\begin{aligned}
E_{\Delta}^{z}(\rho) & =\int_{1-\Delta}^{1} x^{2}\left(1-x^{2}\right)^{-1 / 4} J_{0}(k \rho x) \mathrm{d} x \\
& \approx \frac{\Delta(1-\Delta / 2)^{2}}{\left[1-(1-\Delta / 2)^{2}\right]^{1 / 4}} J_{0}\left(k \rho\left(1-\frac{\Delta}{2}\right)\right) .
\end{aligned}
$$

Thus, similar to the scalar case (see (15)), the focal spot size is determined by the first zero of the zero-order Bessel function. Hence, with decreasing annular pupil's width $\Delta \rightarrow$ 0 , we obtain in the limit

$$
\rho_{\text {min }}^{z \text {,ring }}=\frac{0.38}{\mathrm{NA}} \lambda \underset{\mathrm{NA} \rightarrow 1}{\longrightarrow} \rho_{\lim }^{z \text {,ring }}=0.38 \lambda .
$$

The focal spot size can be further decreased without an essential side-lobe increase based on the destructive interference if a $\pi$-radian phase jump is introduced along the central radius of the narrow annular aperture (16). Then, the field distribution in the focal plane is given by

$$
\begin{aligned}
E_{\tau}^{z}(\rho)= & \int_{1-\Delta}^{1-\Delta / 2} x^{2}\left(1-x^{2}\right)^{-1 / 4} J_{0}(k \rho x) \mathrm{d} x \\
& -\int_{1-\Delta / 2}^{1} x^{2}\left(1-x^{2}\right)^{-1 / 4} J_{0}(k \rho x) \mathrm{d} x .
\end{aligned}
$$

To calculate the integrals in (28), we write down

$$
\begin{aligned}
E_{\tau}^{z}(\rho) \approx & J_{0}\left(k \rho\left(1-\frac{3 \Delta}{4}\right)\right) \frac{1}{(1-3 \Delta / 4)} \\
& \times\left.\left[-\frac{2}{3} x^{2}\left(1-x^{2}\right)^{3 / 4}-\frac{8}{21}\left(1-x^{2}\right)^{7 / 4}\right]\right|_{1-\Delta} ^{1-\Delta / 2} \\
& -J_{0}\left(k \rho\left(1-\frac{\Delta}{4}\right)\right) \frac{1}{(1-\Delta / 4)} \\
& \times\left.\left[-\frac{2}{3} x^{2}\left(1-x^{2}\right)^{3 / 4}-\frac{8}{21}\left(1-x^{2}\right)^{7 / 4}\right]\right|_{1-\Delta / 2} ^{1}
\end{aligned}
$$

Then, retaining the $\Delta$ terms up to the second order, we obtain

$$
\begin{aligned}
E_{\tau}^{z}(\rho) \approx-\Delta^{3 / 4}\{( & \left.0.213+0.386 \Delta-1.139 \Delta^{2}\right) \\
& \left.\quad-\frac{k^{2} \rho^{2}}{4}\left(0.213+0.734 \Delta-2.175 \Delta^{2}\right)\right\} .
\end{aligned}
$$

From (30) an estimate for the focal spot radius then takes the form

$$
\rho_{z, \mathrm{jump}} \approx \frac{\lambda}{\pi} \sqrt{\frac{0.213+0.386 \Delta-1.139 \Delta^{2}}{0.213+0.734 \Delta-2.175 \Delta^{2}}},
$$

with the minimum attained at $\Delta_{m}=0.168$.

Thus, the minimal estimate of the central spot radius is given by

$$
\rho_{z, \mathrm{jump}}\left(\Delta_{m}\right) \approx 0.301 \lambda
$$

\section{Numerical Results}

Although the estimates discussed in Section 3 are approximate, they enable us to analyze in which way the focal spot 


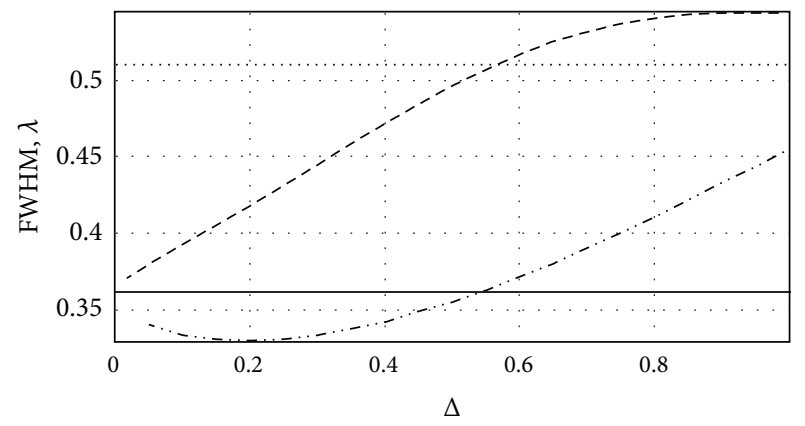

FIgURE 2: The focal spot size (FWHM) as a function of the annular aperture's width $(\Delta)$ without (dashed line) and with (dashed-dotted line) a radial phase jump.

size and intensity depend on the annular aperture's width. For analytical calculations, it was convenient to use the middle radius of the ring. We understand that the radius of phase jump is additional parameter for optimization of the focal distribution; however, experience of other researchers [4, 6$8,19-21]$ shows that even a plenty of free parameters does not allow to reduce the focal spot simultaneously keeping energy. Therefore, we have decided to vary only the width of the ring with the middle $\pi$-phase jump. This limitation may be convenient for the experimental realization.

In this section, the sharp focusing of a radially polarized beam is numerically simulated using the Richards-Wolf integral relations [23] for an aplanatic focusing system.

Figure 2 shows the focal spot size (FWHM) as a function of the annular aperture width $(\Delta)$ for an element without and with the radial phase jump of (16). The dotted horizontal line denotes the scalar limit for the lens $(\mathrm{FWHM}=0.51 \lambda)$, whereas the solid line denotes the scalar limit for the infinitely narrow ring $(\mathrm{FWHM}=0.36 \lambda)$.

Figure 2 suggests that with a simple annular aperture, to go beyond the lens scalar limit, it is necessary to block a lens central portion of radius $0.43 R(\Delta=0.57)$, which amounts to $\sim 20 \%$ of the lens area. Note that all $E$-field components are accounted for in the total intensity. Meanwhile, the results corresponding to the infinitely narrow aperture (FWHM $=$ $0.36 \lambda$ ) remain unattained even at a very small width (at $\Delta=$ $0.01, \mathrm{FWHM}=0.367 \lambda$ ).

If there is a radial phase jump (16), there is no need to block any part of the lens to go beyond the scalar limit. At the same time, blocking the central rays on the radius $0.46 R(\Delta=$ 0.54 , with $\sim 20 \%$ of the area got covered) enables obtaining a focal spot smaller than the scalar limit for the infinitely narrow ring.

Note that the minimal size of FWHM $=0.329 \lambda$ is attained when the annular aperture's width is $\Delta_{m}=0.2$ (Figure 2). This value is approximately 1.2 times larger than the spot size derived in the previous section and employed in the estimate of (32).

Figure 3 shows the plot of the normalized focal point intensity against the annular aperture's width $(\Delta)$ without and with a radial phase jump. The vertical dotted lines mark the annular aperture's width at which the focal spot of size FWHM $=0.38 \lambda$ is formed: for a conventional annular

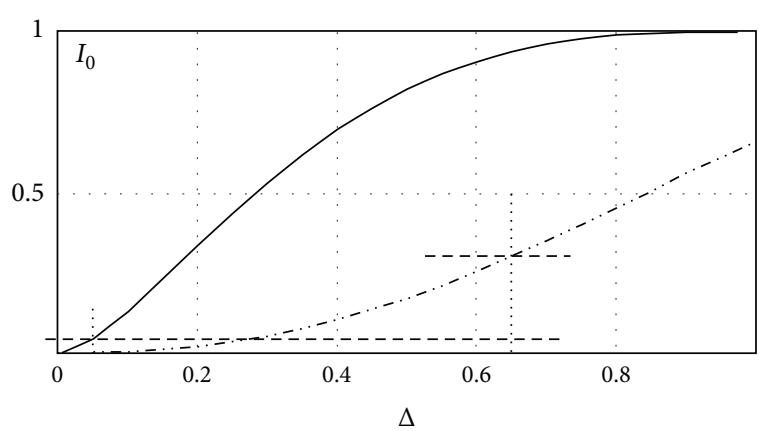

FIGURE 3: Normalized focal point intensity as a function of the annular aperture's width $(\Delta)$ without (solid bold line) and with a radial phase jump (dotted line).

aperture the width is $\Delta=0.06$, and for aperture with the introduced phase jump the width is $\Delta=0.65$. Ring size of $\Delta=0.06$ is rather narrow. Thus, for practical reasons, we decided to start from this value rather than the limit of FWHM $=0.36 \lambda$. It is seen that when the phase jump was introduced, the focal point intensity generated with the wider annular aperture is several times larger than that generated with the conventional narrow ring.

Table 1 gives the numerical simulation results when a radially polarized beam is tightly focusing $(R=100 \lambda, f=$ $101 \lambda$ ) and a narrow annular aperture with the phase jump of (16) is used: $\Delta$ is the annular slit width, $I_{0}$ is the total intensity in the focus, $S$ is the ratio of the total intensity of the first side lobe to that of the central focal spot, FWHM is the central focal spot diameter in terms of full-width halfmaximum intensity, and $\mathrm{FWHM}_{z}$ is the central spot diameter at half-maximum intensity of the longitudinal component.

Table 1 suggests that the smallest central focal spot (FWHM $=0.329 \lambda$, see 2nd row of Table 1) has been attained without an unacceptable increase in the side lobes $(S<$ $0.3)$. However, there is an essential loss of the central spot energy, with the intensity $I_{0}$ being half of that provided by the narrow annular aperture (1st row of Table 1). The longitudinal distribution suggests that the focal spot becomes tighter due to the destructive interference of the beams transmitted by different aperture's rings.

However, by increasing the pupil's width, the central spot energy can be significantly increased with spot size smaller than that generated with the narrow ring. In particular, at $\Delta=0.6$ (3rd row of Table 1 ), the focal spot is slightly smaller (FWHM $=0.371 \lambda)$, whereas the intensity is $\sim 6$ times larger when compared with that generated by the narrow annular aperture. A disadvantage consists in somewhat higher side lobes $(S=0.35)$.

For the full-aperture case with $\pi$-phase jump $(\Delta=1,4$ th row of Table 1), what we observe is not only a larger focal intensity but also lower side lobes. In this case, the focal spot size $(\mathrm{FWHM}=0.454 \lambda)$ does not exceed the Airy pattern's scalar limit.

Results for the case of full-aperture illumination without any apodization are shown (5th row of Table 1) for comparison: it is possible to see that the focal spot size (FWHM = $0.544 \lambda$ ) exceeds the Airy pattern's scalar limit. 
TABLE 1: Numerical simulation results.

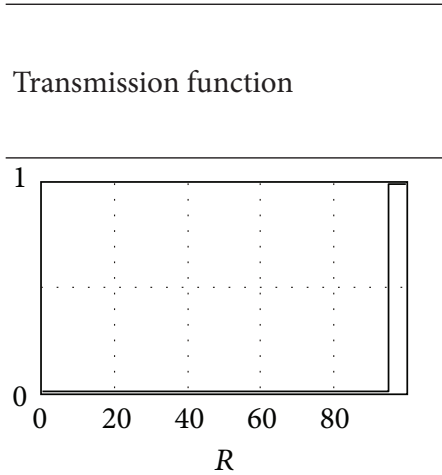

Ring, $\Delta=0.05$

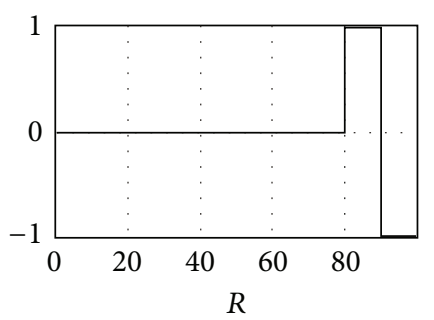

Jump, $\Delta=0.2$

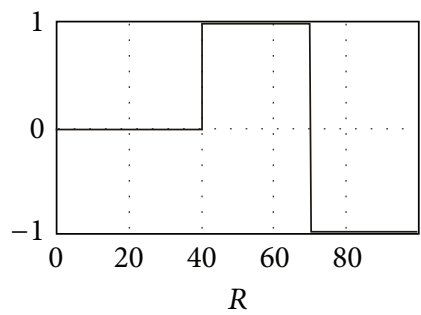

Jump, $\Delta=0.6$

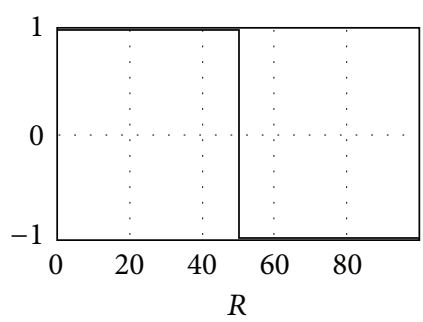

Jump, $\Delta=1$

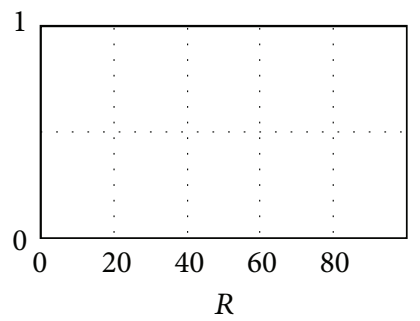

Full-aperture illumination

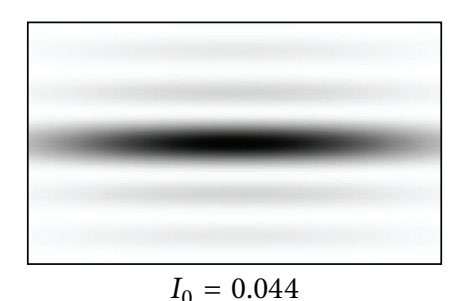

$I_{0}=0.044$

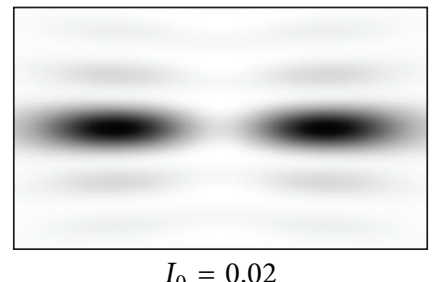

$I_{0}=0.02$

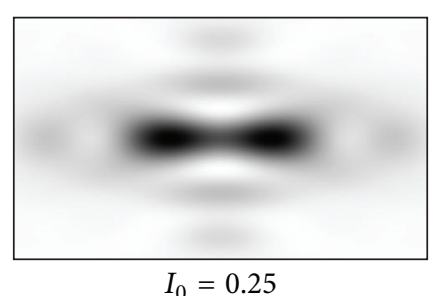

$I_{0}=0.25$

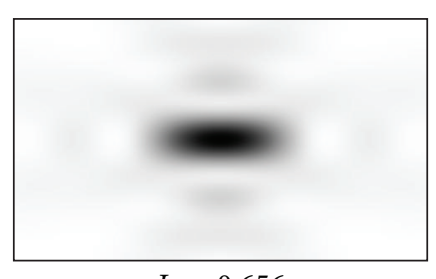

$I_{0}=0.656$

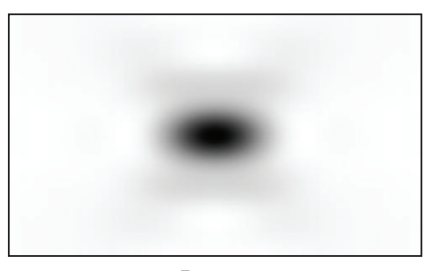

$I_{0}=1$
Transverse intensity distribution in the focus (negative image), $3 \lambda \times 3 \lambda$

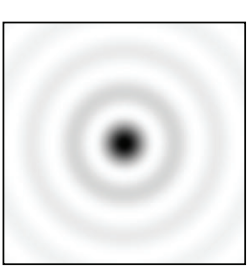

$S=0.162$

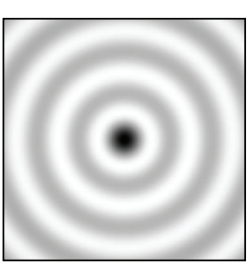

$S=0.279$

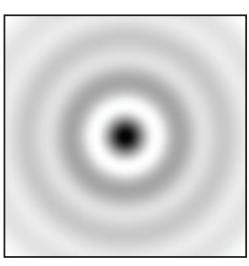

$S=0.351$

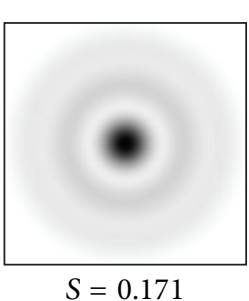

$S=0.171$

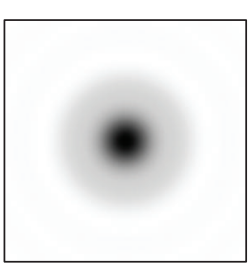

$S=0.16$
Intensity profiles for the field components in the focus (dashed line: transverse; solid line: longitudinal; dotted line: total)

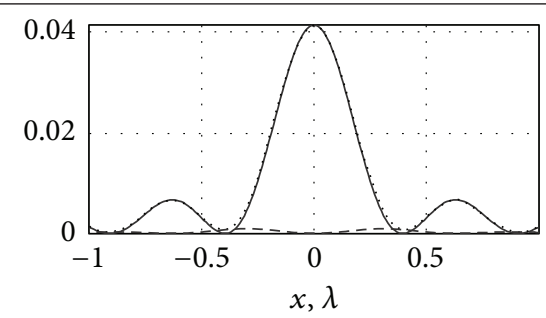

$\mathrm{FWHM}=0.378 \lambda, \mathrm{FWHM}_{z}=0.372 \lambda$

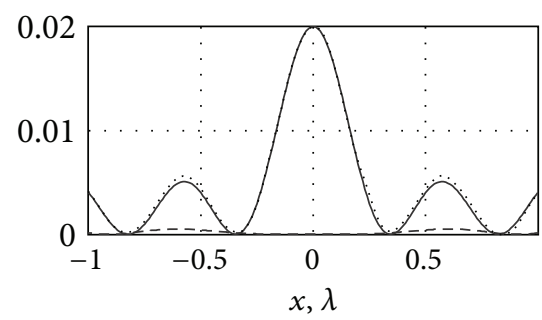

$\mathrm{FWHM}=0.329 \lambda, \mathrm{FWHM}_{z}=0.329 \lambda$

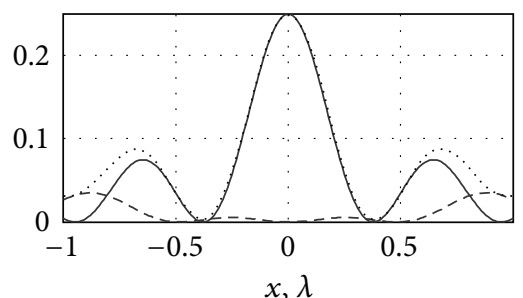

$\mathrm{FWHM}=0.371 \lambda, \mathrm{FWHM}_{z}=0.363 \lambda$

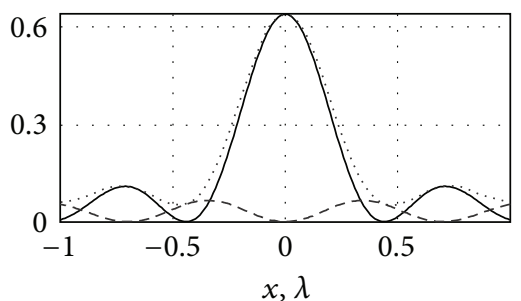

$\mathrm{FWHM}=0.454 \lambda, \mathrm{FWHM}_{z}=0.412 \lambda$

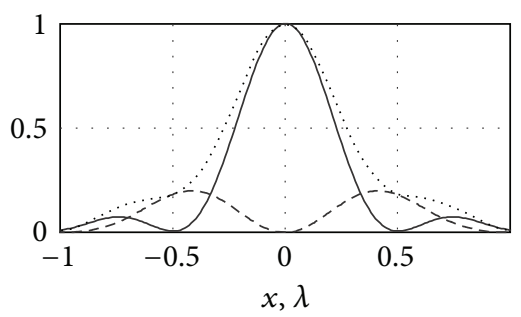

$\mathrm{FWHM}=0.544 \lambda, \mathrm{FWHM}_{z}=0.45 \lambda$

\section{Conclusions}

The analysis of the sharp focusing has shown that the dependence of the focal spot size on the narrow annular pupil's width is different for the scalar and vector models.
In the scalar model, when describing the focusing based on the Fourier transform, the smallest focal spot is attained when the peripheral ring width tends to zero, while the focus intensity drops quadratically with decreasing ring width. 
In the vector model of the sharp focusing of the radially polarized beam (Debye's approximation), the smallest focal spot is obtained at a fixed annular aperture's width equal to $20 \%$ of the full-aperture radius. The amplitude at the focus is proportional to the ring width to the power $3 / 4$. It is one of the most important analytical results which allows us to estimate the energy that is stored in a central spot.

It has been analytically and numerically shown that the destructive interference between the beams passing differentphase rings enables going beyond a scalar limit corresponding to the zero-order Bessel function (FWHM $=0.36 \lambda)$. A tighter focal spot $(\mathrm{FWHM}=0.33 \lambda)$ is attained at a sacrifice of a lower focal intensity and higher side lobes, with the latter not becoming larger than $30 \%$ of the central peak.

We have numerically shown that by introducing a radial $\pi$-phase jump along the aperture's middle radius the scalar limit can be broken: we have got FWHM $=0.454 \lambda$ (for the Airy pattern FWHM $=0.51 \lambda)$. In the meantime, blocking out the central rings with a $0.46 R$ stop (covering $\sim 20 \%$ of the area) enables obtaining a smaller focal spot $(\mathrm{FWHM}=0.371 \lambda)$ with intensity nearly 6 times higher than for simple narrow ring. The lack is higher side-lobes reaching up to $35 \%$ of the central peak intensity.

Certainly, reduction of the focal spot size just by factor 1.65 (from FWHM $=0.544 \lambda$ for open aperture to FWHM = $0.329 \lambda$ for ring with jump) is accompanied with huge losses of energy in this spot (by factor 50). It is a significant disadvantage for the applications based on high energy such as a nonlinear scanning microscopy. Nevertheless, optical microscopy applications can tolerate very high losses: scanning microscopes can work with only a few photons per second providing sensitivity to tens orders below of laser power [11].

\section{Acknowledgments}

The work was financially supported by the Ministry of Education and Science of the Russian Federation (Project 8231) and by the Russian Foundation for Basic Research (Grants 13-07-00266 and 13-07-97004-r_povolzhe_a).

\section{References}

[1] S. Quabis, R. Dorn, M. Eberler, O. Glöckl, and G. Leuchs, "Focusing light to a tighter spot," Optics Communications, vol. 179, no. 1, pp. 1-7, 2000.

[2] R. Dorn, S. Quabis, and G. Leuchs, "Sharper focus for a radially polarized light beam," Physical Review Letters, vol. 91, no. 23, pp. 2339011-2339014, 2003.

[3] C. J. R. Sheppard and A. Choudhury, "Annular pupils, radial polarization, and superresolution," Applied Optics, vol. 43, no. 22, pp. 4322-4327, 2004.

[4] L. E. Helseth, "Mesoscopic orbitals in strongly focused light," Optics Communications, vol. 224, no. 4-6, pp. 255-261, 2003.

[5] Y. Kozawa and S. Sato, "Sharper focal spot formed by higherorder radially polarized laser beams," Journal of the Optical Society of America A, vol. 24, no. 6, pp. 1793-1798, 2007.
[6] C.-C. Sun and C.-K. Liu, "Ultrasmall focusing spot with a long depth of focus based on polarization and phase modulation," Optics Letters, vol. 28, no. 2, pp. 99-101, 2003.

[7] H. Wang, L. Shi, B. Lukyanchuk, C. Sheppard, and C. T. Chong, "Creation of a needle of longitudinally polarized light in vacuum using binary optics," Nature Photonics, vol. 2, no. 8, pp. 501-505, 2008.

[8] S. N. Khonina and S. G. Volotovsky, "Controlling the contribution of the electric field components to the focus of a highaperture lens using binary phase structures," Journal of the Optical Society of America A, vol. 27, no. 10, pp. 2188-2197, 2010.

[9] S. N. Khonina, A. V. Ustinov, and E. A. Pelevina, "Analysis of wave aberration influence on reducing focal spot size in a highaperture focusing system," Journal of Optics, vol.13, no. 9, Article ID 095702, 2011.

[10] G. Toraldo di Francia, "Degrees of freedom of an image," Journal of the Optical Society of America A, vol. 59, no. 7, pp. 799-804, 1969.

[11] F. M. Huang and N. I. Zheludev, "Super-resolution without evanescent waves," Nano Letters, vol. 9, no. 3, pp. 1249-1254, 2009.

[12] T. R. M. Sales and G. M. Morris, "Diffractive superresolution elements," Journal of the Optical Society of America A, vol. 14, no. 7, pp. 1637-1646, 1997.

[13] S. N. Khonina and I. Golub, "Enlightening darkness to dif fraction limit and beyond: comparison and optimization of different polarizations for dark spot generation," Journal of the Optical Society of America A, vol. 29, no. 7, pp. 1470-1474, 2012.

[14] J. Bewersdorf, A. Egner, and S. W. Hell, "4pi-confocal microscopy is coming of age," Imaging \& Microscopy, vol. 4, pp. 24-25, 2004.

[15] L. E. Helseth, "Breaking the diffraction limit in nonlinear materials," Optics Communications, vol. 256, no. 4-6, pp. 435438, 2005.

[16] N. Bokor and N. Davidson, "Tight parabolic dark spot with high numerical aperture focusing with a circular $\pi$ phase plate," Optics Communications, vol. 270, no. 2, pp. 145-150, 2007.

[17] V. V. Kotlyar, S. N. Khonina, A. S. Melekhin, and V. A. Soifer, "Fractional encoding method for spatial filters computation," Asian Journal of Physics, vol. 8, no. 3, pp. 273-286, 1999.

[18] S. N. Khonina, S. A. Balalayev, R. V. Skidanov, V. V. Kotlyar, B. Päivänranta, and J. Turunen, "Encoded binary diffractive element to form hyper-geometric laser beams," Journal of Optics A, vol. 11, no. 6, Article ID 065702, 7 pages, 2009.

[19] W. Chen and Q. Zhan, "Three-dimensional focus shaping with cylindrical vector beams," Optics Communications, vol. 265, no. 2, pp. 411-417, 2006.

[20] X. Gao, J. Wang, H. Gu, and W. Xu, "Focusing properties of concentric piecewise cylindrical vector beam," Optik, vol. 118, no. 6, pp. 257-265, 2007.

[21] K. Huang, P. Shi, X.-L. Kang, X. Zhang, and Y. P. Li, “Design of DOE for generating a needle of a strong longitudinally polarized field," Optics Letters, vol. 35, no. 7, pp. 965-967, 2010.

[22] B. Tian and J. Pu, "Tight focusing of a double-ring-shaped, azimuthally polarized beam," Optics Letters, vol. 36, no. 11, pp. 2014-2016, 2011.

[23] B. Richards and E. Wolf, "Electromagnetic diffraction in optical systems. II. Structure of the image field in an aplanatic system," Proceedings of the Royal Society A, vol. 253, no. 1274, pp. 358379, 1959. 
[24] M. Abramowitz and I. A. Stegun, Handbook of Mathematical Functions, Courier Dover Publications, North Chelmsford, Mass, USA, 1972. 

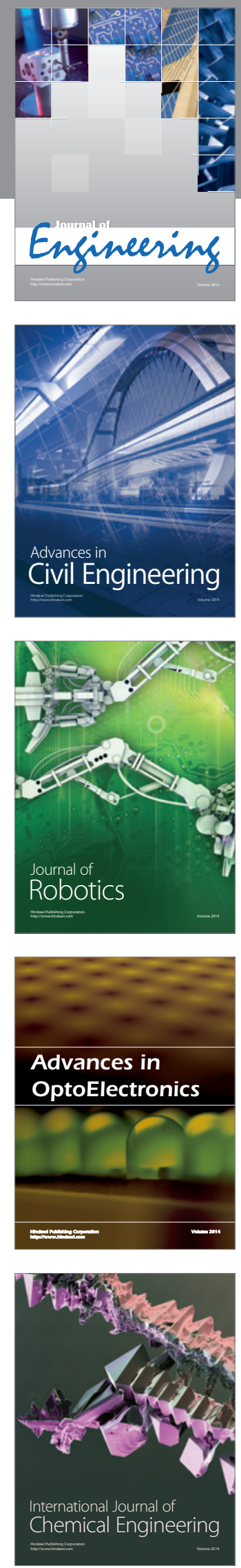

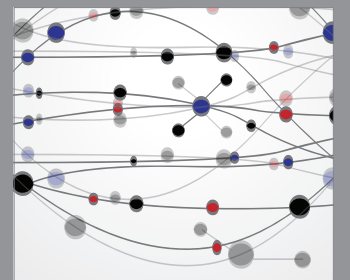

The Scientific World Journal
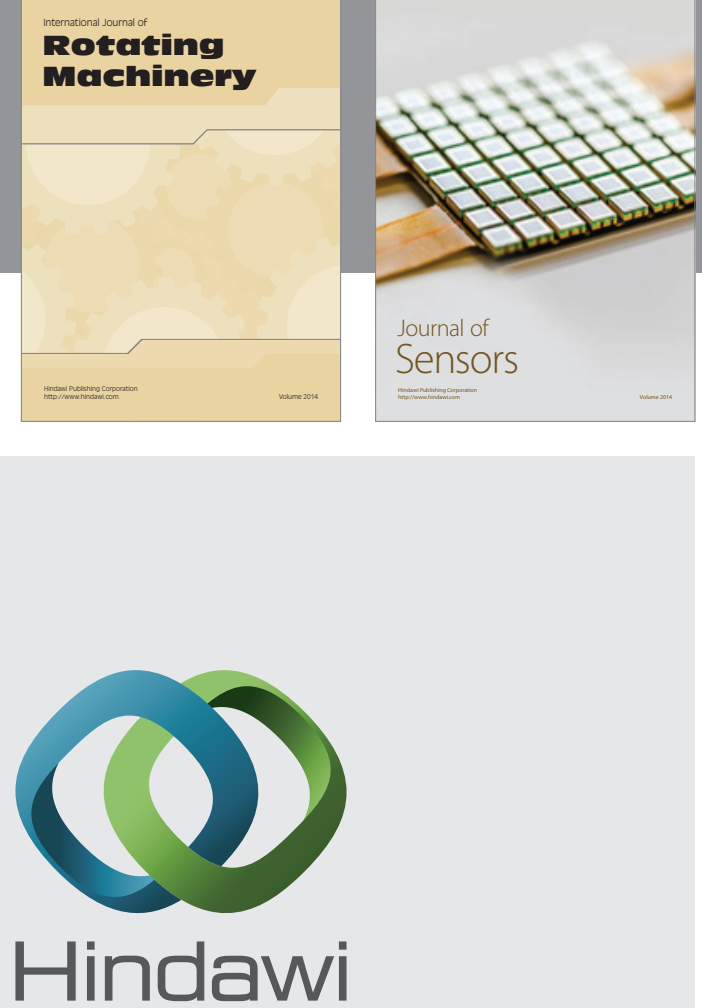

Submit your manuscripts at http://www.hindawi.com
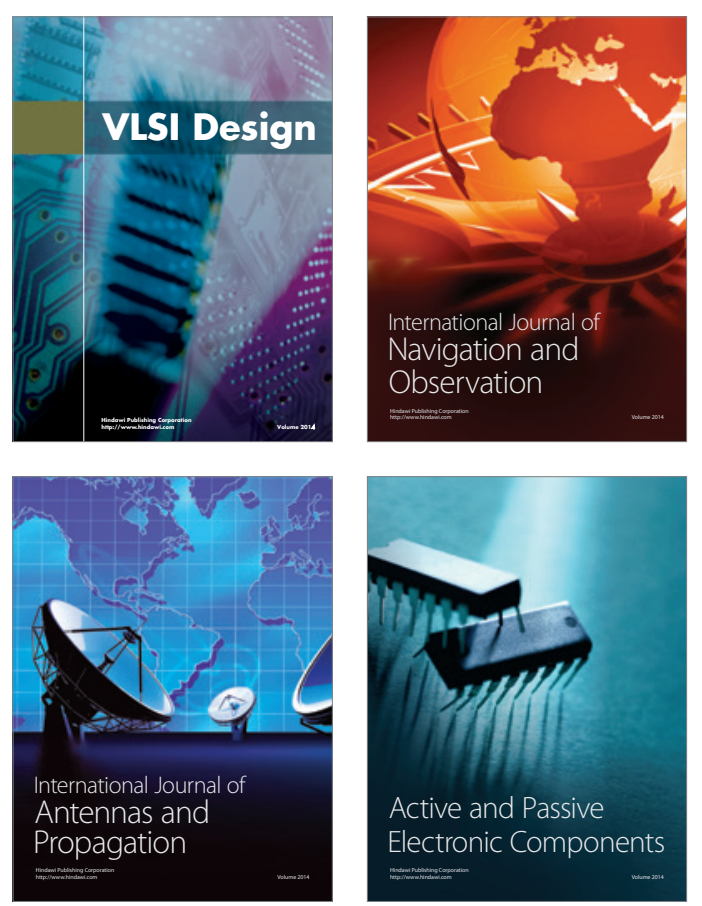
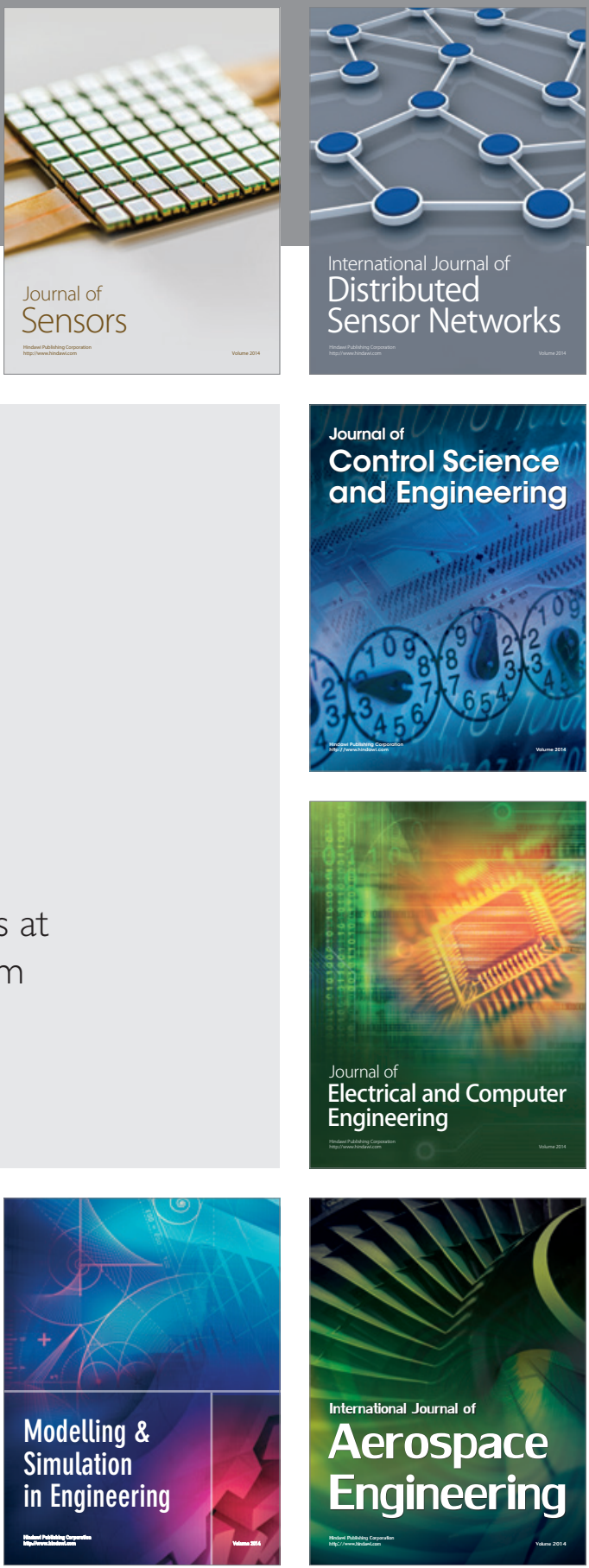

Journal of

Control Science

and Engineering
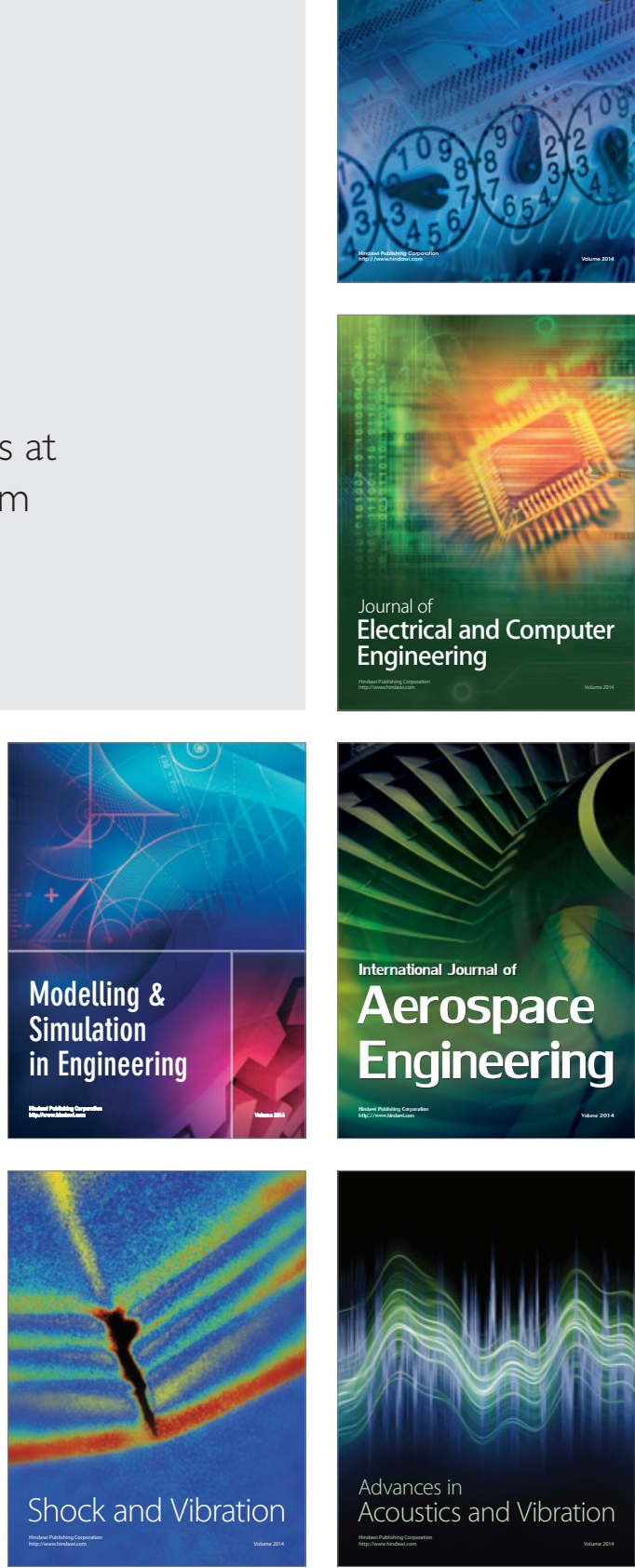